\title{
The Effect of Intravenous Staphylococcal Enterotoxin B Intoxication on Physiological Parameters in Rhesus Macaques As Measured By Telemetry
}

\author{
Daniel W Christensen ${ }^{1}$, Nancy J Keller ${ }^{1}$, Yael G Snyder ${ }^{2}$, William D Pratt ${ }^{3}$, Franco Rossi ${ }^{3}$, David P Fetterer ${ }^{4}$, Luis \\ Lugo Roman ${ }^{1}$ and Bret K Purcell ${ }^{2}$
}

${ }^{1}$ Veterinary Medicine Division, United States Army Medical Research Institute of Infectious Diseases, USA

${ }^{2}$ Bacteriology Division, United States Army Medical Research Institute of Infectious Diseases, USA

${ }^{3}$ Virology Division, United States Army Medical Research Institute of Infectious Diseases, USA

${ }^{4}$ Biostatistics Division, United States Army Medical Research Institute of Infectious Diseases, USA

Submission: November 09, 2017; Published: November 27, 2017

*Corresponding author: : Daniel W Christensen, Veterinary Medicine Division, United States Army Medical Research Institute of Infectious Diseases, Frederick, USA; Email: daniel.w.christensen14.mil@mail.mil

\begin{abstract}
Staphylococcal enterotoxin B (SEB) is a super antigen implicated in food borne illness, toxic shock syndrome, and potential use as a biowarfare agent. Continuous monitoring of physiological data during SEB intoxication has largely been limited to temperature in previous studies. In an effort to expand the current database of knowledge and validate the rhesus macaque model, radiotelemetry devices were implanted into three rhesus macaques, and multiple physiological parameters were monitored in real time after an intravenous intoxication of SEB. Statistically significant changes were noted for blood pressure, heart rate, respiration rate, temperature, and ECG values. These trends may be used as a baseline for future studies involving SEB intoxication in the rhesus macaque.
\end{abstract}

Keywords: Nonhuman Primate; Telemetry; Staphylococcal Enterotoxin B

Abbreviations: TSS: Toxic Shock Syndrome; SEB: Staphylococcal Enterotoxin B; TSS: Toxic Shock Syndrome; LVP: left Ventricular Pressure; FDA Federal Drug Administration; AOP: Aortic Pressure; CDC: Center for Disease Control;

\section{Introduction}

Staphylococcal Enterotoxin B (SEB) is a superantigen globally responsible for numerous foodborne illness outbreaks [1-4]. In a recent study [5], SEB was estimated to cause 9.4 million episodes of foodborne illness per year in the United States. In a 2013 report from the European Union [6], bacterial toxins from Staphylococcus, Bacillus, and Clostridium were the third most leading cause of foodborne illness outbreaks where the agent was known, implicated in 834 outbreaks resulting in 9,203 cases with 452 hospitalizations. SEB is also one of the leading causes of toxic shock syndrome (TSS) [7], and has been described as a potential biowarfare agent [8].

Individual case reports and published outbreak investigations list symptoms observed after intoxication. These symptoms vary dependent on the route of exposure. For gastrointestinal exposure, common symptoms include nausea, vomiting, abdominal pain, cramps and diarrhea with an onset of minutes to hours after intoxication [1-4,9]. After mucocutaneous exposure, moderate to severe conjunctival symptoms were noted, with or without concurrent gastrointestinal symptoms, upper respiratory symptoms, and/or fever with an onset of 1-9 hrs $[4,10]$. Inhalational exposure resulted in lower respiratory symptoms, myalgia, gastrointestinal symptoms, and fever. The onset of symptoms was from 1.5-24 hours $[4,10]$. Systemic exposure, most commonly leading to TSS, includes symptoms of fever, rash, hypotension, tachycardia, multiorgan failure, and desquamation of the hands and feet, with or without concurrent gastrointestinal symptoms. Systemic intoxication can be from both menstrual and non-menstrual origin of infection [7,11-14].

The Animal Rule, as defined by the Federal Drug Administration (FDA), stipulates that well-controlled animal studies may replace human challenge studies in cases where 
the agent involved cannot be ethically or feasibly administered to humans. SEB is classified by the Center for Disease Control (CDC) as a select agent under the Federal Select Agent Program, and as such is often used in animal studies for the development of therapeutic countermeasures. SEB has been previously modeled in the rhesus macaque (Macacamulatta) with success for intoxication [15-22] and is the best model for lung pathology following inhalational exposure [23-25]. However, not much is known about the physiological parameters in the rhesus macaque model to evaluate the similarities compared to human intoxication.

An important consideration for all studies involving animals is the three R's of reduction, replacement, and refinement [2628]. Radiotelemetry devices allow for continuous collection of multiple data points, thus allowing for an increase in the amount of data that may be collected from one animal. This increase in data can then potentially facilitate the overall reduction in animal numbers needed for a study, while still maintaining statistical relevance. This is especially useful for studies involving SEB, where the pathophysiology is complex and multiple physiological parameters are involved [1-4]. Real-time analysis of these parameters can further elucidate the pathophysiology of SEB and potentially identify therapeutic windows where intervention is likely to achieve maximum effect. Several species of animals have been used in SEB studies, but telemetry data in these studies has been solely limited to the collection of body temperature $[2,17,28-30]$.

The first objective of this study was to establish a baseline of physiological parameters for the rhesus macaque using radiotelemetry devices after intravenous injection of SEB. The second objective of the study was to determine whether the physiological data from the rhesus macaque model for SEB intoxication aligns with the observed human intoxication syndromes, supporting or refuting its validity as a model.

\section{Materials and Methods}

\section{Non-Human Primates and Surgery}

Research was conducted under an IACUC approved protocol in compliance with the Animal Welfare Act, PHS Policy, and other Federal statutes and regulations relating to animals and experiments involving animals [31,32]. The facility where this research was conducted is accredited by the Association for Assessment and Accreditation of Laboratory Animal Care, International and adheres to principles stated in the Guide for the Care and Use of Laboratory Animals [31], National Research Council, 2011. Three (1M, 2F) healthy adult (>5yr) rhesus macaques were obtained from commercial vendors and evaluated for normal health and behavior prior to study initiation. All macaques were surgically implanted with T27F-1B radiotelemetry devices (Konigsberg Instruments, Inc., Pasadena, CA) at least one year prior. All macaques were then surgically implanted with two central venous catheters, placed on either side of the neck, in the jugular veins. These catheters were then tethered to the outside of their cages via a jacket and tether system. The macaques were allowed sufficient time to acclimate to the jackets and tethers prior to surgery.

Three macaques $(n=3)$ received a $50 \mu$ dose of SEB intravenously through one of the tethered catheters. SEB was purchased from Toxin Technology, Inc. (Sarasota, FL, Lot 92109B) and rehydrated in PBS (Sigma, P3813, Lot 031M8214) at a concentration of $5 \mathrm{ug} / \mathrm{kg}$. The intoxications were initiated late at night, and monitored for a period of seven hours. All monitoring took place via telemetry, and room entry was restricted to minimize potential stressors to the macaques.

\section{Telemetric Monitoring}

The implanted radiotelemetry devices allowed for the continuous monitoring of data involving aortic pressure (AOP), left ventricular pressure (LVP), intrathoracic pressure, electrocardiography (ECG), and temperature. The telemetry data were captured and analyzed using the Notocord-hem Evolution software platform (Version 4.3, Notocord Inc., and Newark, NJ). Five-minute averages of the above listed physiologic parameters were calculated for each subject. Telemetry data obtained during the five days prior to SEB administration were used to calculate baseline values, and provided the average and standard deviation (SD) of each five-minute time period of a 24-h day.

\section{Statistical Analysis}

For each telemetry parameter, two-way repeated measures mixed linear model [33], having fixed effects for time post challenge and the hour of the day was fit to the data. The inclusion of hour of the day was necessary to control for the expected diurnal variations in most telemetry parameters. No interaction term was included between the time of day and hour post challenge, so that the effects of time of day and time post challenge were taken to be additive. At each time point post challenge, an estimate of the expected change from pre-challenge values, controlling for the effect of diurnal variations was obtained as an appropriate contrast in the linear model. Denominator degrees of freedom were estimated by Satterwaite's method [34]. Correlations within subjects were modeled as compound symmetric. No adjustment is included for the multiple comparisons across time points. Analysis was implemented in SAS PROC MIXED, SAS version 9.4.

\section{Results}

The five following parameter groups were selected for analysis and comparison: blood pressure (AOP Approx Mean, AOP DIA, AOP Mean, AOP Pulse Press, AOP Quad Mean, AOP SYS, LVP Mean, LVP Systolic, LVP EDP, LV dPdt 40), heart rate (AOP Beat Interval, AOP Rate, Heart Rate, LV Rate), ECG (PR duration, QRS duration, QT duration, QTcBazett, QTcFridericia), respiration rate (Resp Cycle duration, Resp Expiratory time, Respiration Rate), and temperature. Nearly all values showed statistical significance at multiple time points (Table 1). A single parameter will be used from each group to illustrate the trends. 


\section{Journal of Pharmacology \& Clinical Research}

\begin{tabular}{|c|c|c|c|c|c|c|c|c|c|c|c|c|c|c|c|c|}
\hline : & 离 & 高离 & 䊉 & 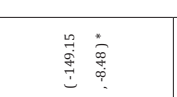 & 号 & 旁: & $\underset{\text { तु }}{\pi}$ & 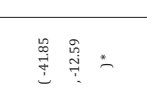 & 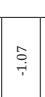 & 姁要 & 足 & 总 & $\dddot{n}$ & 商离 & 蒋 & 苞 \\
\hline : & 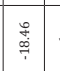 & 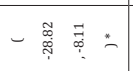 & 啹 & 善㖕: & 商 & 一 & 商 & - 总. & 号 & 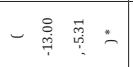 & 总 & 一誉 & $\frac{a}{0}$ & 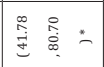 & ‡ู้ & 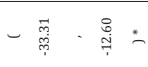 \\
\hline 。 & 旁 & 总 & 蛋 & 一葶亭 & 官 & 商 & 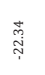 & 范 & $\dddot{\Xi}$ & 害富 & : خै & 窇 & 高 & 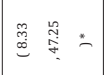 & ๙్ & 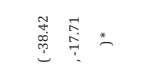 \\
\hline 品 & 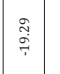 & 亭离 & ֻู & 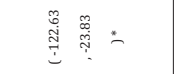 & 憘 & 䒽 & 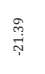 & 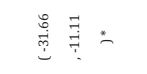 & $\frac{\mathscr{O}}{\square}$ & 密宙 & 富 & 喜营 & \&: & 要: & : & 莴 \\
\hline 品 & 萬 & 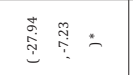 & 爱 & 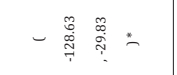 & 莘 & 总青 & 乫 & 总毒: & 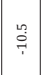 & 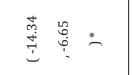 & 旁 & 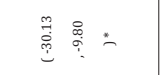 & 落 & 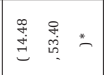 & 乎 & 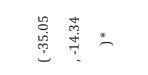 \\
\hline 品 & 誉 & 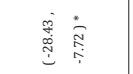 & กั & 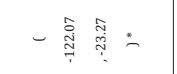 & $\frac{\vec{v}}{\square}$ & 善票 & ָูँ & 衰富 & 总 & 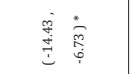 & $\frac{a^{2}}{2}$ & 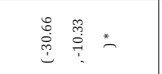 & 僖 & 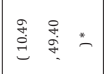 & 岁 & 喜尊 \\
\hline n & 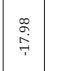 & 夢密 & 旁 & 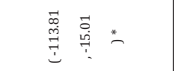 & 号 & 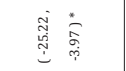 & هั: & 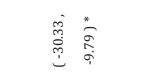 & 亭 & 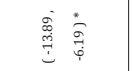 & 愛 & 总号 & $\frac{9}{3}$ & 言密 & 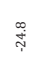 & 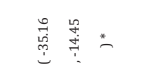 \\
\hline$\stackrel{n}{*}$ & 誉 & 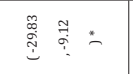 & 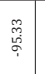 & 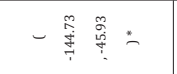 & 蒿 & 雚 & $\stackrel{m}{\vec{q}}$ & 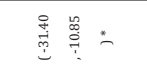 & 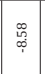 & $\stackrel{2}{2}$ & \begin{tabular}{|l|l}
$\frac{9}{7}$ \\
\end{tabular} & 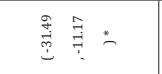 & 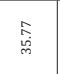 & 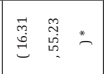 & 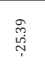 & 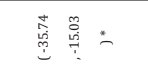 \\
\hline ? & 莡 & 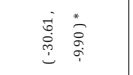 & 孚 & 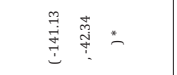 & : & 密 & 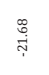 & 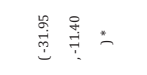 & 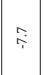 & 㝵富 & 惫 & 帯 & 器 & 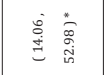 & 悹 & 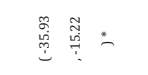 \\
\hline$\stackrel{\not}{a}$ & 尊 & 商衰 & $\overrightarrow{\tilde{q}}$ & 一蛋蒡 & 愛 & 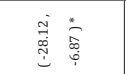 & 今్ จे & 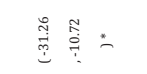 & 帝 & 宰 & 宵 & 总总 & 嘉 & 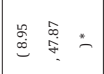 & 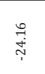 & 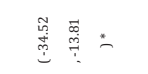 \\
\hline . & 疍 & 商 & 墒 & 一箩虽 & 总 & 啇㝘 & 监 & 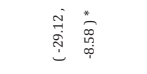 & 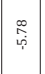 & 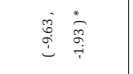 & 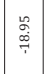 & 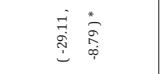 & 烔 & 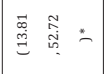 & $\stackrel{\text { }}{\vec{~}}$ & 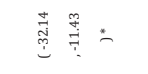 \\
\hline 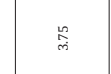 & 总 & 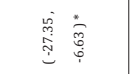 & 胥 & 学 & 㔛 & 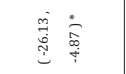 & F & 衰高 & $\stackrel{\wp}{q}$ & 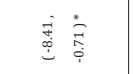 & ֶֻ) & 高客 & : & 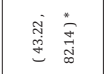 & 薃 & 裹螎 \\
\hline$\stackrel{n}{n}$ & $\frac{z}{y}$ & 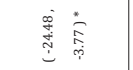 & 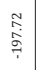 & 善尊: & $\frac{\vec{p}}{\vec{p}}$ & 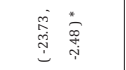 & $\stackrel{2}{\dddot{q}}$ & 急嵩 & $\frac{\approx}{\partial}$ & 絁営 & 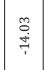 & 产畺 & g్ & 实高 & बुg & 商宙 \\
\hline 品 & 索 & 高 & 虽 & 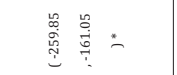 & $\stackrel{\circ}{\%}$ & 要 & \%ั & 商豆 & $\stackrel{g}{~}$ & 总 & $\%$ & 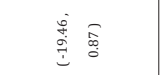 & 赵 & 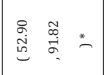 & 莘 & 苞高 \\
\hline m & $\vec{t}$ & 容 & 蔦 & 总总 & 品 & 窎衰 & $\stackrel{\Xi}{\exists}$ & 窎 & $\stackrel{g}{q}$ & 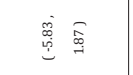 & $\stackrel{\overbrace{}}{\rightrightarrows}$ & 急豈 & 寿 & 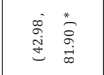 & 冚 & 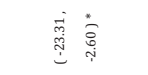 \\
\hline$\stackrel{n}{N}$ & 客 & 高 & 桨 & 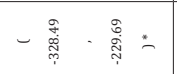 & $\frac{2}{9}$ & 管 & $\stackrel{\circ}{\circ}$ & 荳 & $\stackrel{y}{y}$ & 旁孚 & 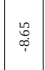 & 尊哥 & 㩊 & 总善 & $\stackrel{n}{g}$ & 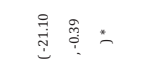 \\
\hline 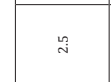 & 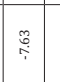 & 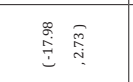 & 品 & 一总 & : & 蛋 & 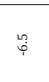 & 商 & 品 & 葛票 & $\overrightarrow{\vec{\varphi}}$ & 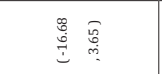 & 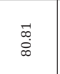 & 总言: & 葛 & 产 \\
\hline 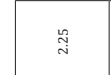 & 趸 & 蹒 & 学 & 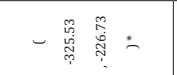 & $\underset{⿱ 乛}{\vec{b}}$ & 突 & 尊 & 离商 & 总 & 高离 & $\vec{p}$ & 高覃 & $\stackrel{m}{n}$ & 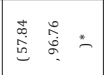 & : & 言 \\
\hline$\sim$ & $\vec{a}$ & 管 & 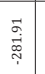 & 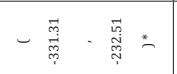 & $\underset{n}{n}$ & 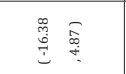 & $\stackrel{q}{q}$ & 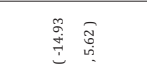 & $\Im$ & 営票 & $\stackrel{q}{q}$ & 啜奇 & 蓄 & 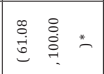 & ֻั & 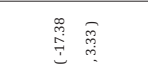 \\
\hline$\stackrel{n}{g}$ & $\frac{q}{q}$ & 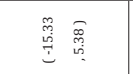 & ฟั. & 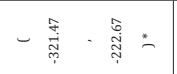 & 舑 & 管㺃 & 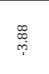 & 美璦 & 密 & 总 & \% & 喜总 & $\stackrel{n}{\alpha}$ & 善器二 & 草 & 总 \\
\hline 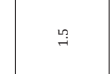 & $\frac{8}{6}$ & 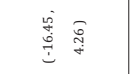 & 总 & 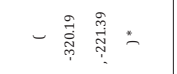 & $\stackrel{F}{q}$ & 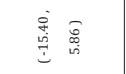 & $\stackrel{g}{\partial}$ & 㩊要 & 辤 & 惢 & $\frac{\substack{p \\
p}}{b}$ & 总高 & $\stackrel{\infty}{\sigma}$ & 善嘉 & 总 & 总要 \\
\hline$\stackrel{乛}{\sharp}$ & $\dddot{F}$ & 寻 & 总 & 一品 & $\stackrel{q}{q}$ & 愛 & $\dddot{\varpi}$ & 号高 & \% & 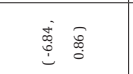 & $\stackrel{p}{q}$ & 尌要 & : & 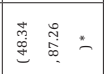 & $\tilde{q}$ & 絰要 \\
\hline- & $\frac{\partial}{\partial}$ & 商爵 & 节 & 一蒡蒡 & 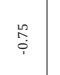 & 䙵票 & $\stackrel{8}{\square}$ & 高哥 & $:$ & 善要 & 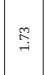 & 实要 & 誊 & 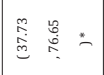 & 㩊 & 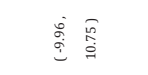 \\
\hline 品 & $\vdots$ & 窟 & 营 & 一憘喜 & : & 要要 & $\frac{\text { a }}{9}$ & 兽 & i & 量要 & à & 高蛋 & స్ల్ & 虽总: & 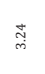 & 䌉 \\
\hline 品 & 菅 & 商离 & 哭 & 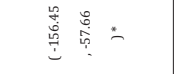 & \%ั & 蒦需 & ?: & 苞垔 & $\sim$ & 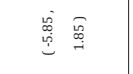 & 触 & 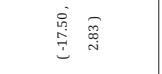 & $\stackrel{8}{=}$ & 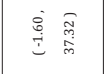 & ळे & 㞭焉 \\
\hline : & $\stackrel{q}{q}$ & 总要 & 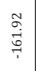 & 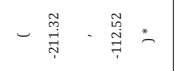 & $\vec{q}$ & 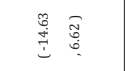 & $\stackrel{n}{p}$ & 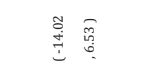 & $\stackrel{8}{q}$ & 裹票 & م. & 虽哑 & 骠 & 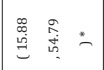 & 品 & 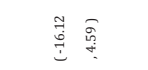 \\
\hline 善 & 产 & 商 & 产 & 产 & $\frac{1}{\frac{1}{2}}$ & 产 & 衰 & 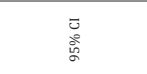 & 言旁 & 商 & $\frac{\sqrt[a]{2}}{2}$ & 产 & 彦 & 产 & 产 & 愛 \\
\hline ) & & 亏ัँ & $\overrightarrow{\mathrm{a}}$ & & & : & to & & & ป⿳亠े冖 & & $\frac{\partial}{v}$ & & ఫั & ⿳亠े口犬 & \\
\hline 亭 & & 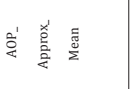 & & 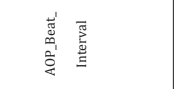 & & 产 & 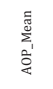 & & & 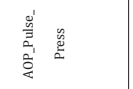 & & 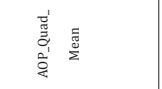 & & 产 & 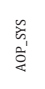 & \\
\hline
\end{tabular}

How to cite this article: Daniel W C, Nancy J K, Yael G S, William D P, Franco R, et al. The Effect of Intravenous Staphylococcal Enterotoxin B Intoxication on Physiological Parameters in Rhesus Macaques As Measured By Telemetry. J of Pharmacol \& Clin Res. 2017; 4(3): 555638. DOI: 10.19080/ JPCR.2017.04.555638. 


\begin{tabular}{|c|c|c|c|c|c|c|c|c|c|c|c|c|c|c|c|c|c|c|c|}
\hline 虽 & 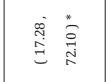 & & & & & 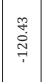 & 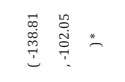 & $\begin{array}{l}\frac{7}{y} \\
\frac{y}{P}\end{array}$ & 鸹㝘 & 彚 & 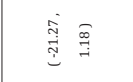 & $\frac{\circ}{9}$ & 总 & $\vec{b}$ & 密禽 & 今 & 宰离 & 帝 & 商 \\
\hline है & 量总: & $\dddot{7}$ & 一䨌高 & $\frac{m^{\prime}}{\vec{b}}$ & 一章孚 & \begin{tabular}{|l|}
\multirow{2}{*}{} \\
\multirow{2}{*}{}
\end{tabular} & 一糢絭: & $\frac{5}{y}$ & 一 总 总: & 获 & 一总虽 & $\vec{े}$ & 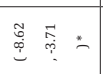 & 喜 & 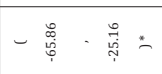 & 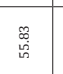 & 青举: & 莒 & 一总 \\
\hline 蒪 & 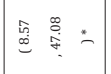 & $\frac{1}{2}$ & 害融 & $\vec{q}$ & 高 & 密 & 高営 & 言 & 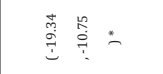 & 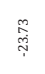 & 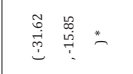 & $\stackrel{\circ}{\circ}$ & 总 & $\overrightarrow{7}$ & 善旁 & $\overrightarrow{5}$ & 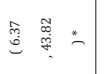 & $\stackrel{7}{7}$ & 一袁焉 \\
\hline 言 & 产 & จุ & 居镸 & 高 & 通亭 & : & 商㩊 & 突 & 旁窝 & 号 & 愛粂: & 商 & 管 & fi & 喜蒡 & 㟥 & 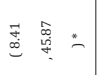 & $\cong$ & 害奇 \\
\hline 商 & 冞芯 & $\dddot{q}$ & 害孚 & $\stackrel{\nexists}{q}$ & 㝘 & : & 咅重 & $\dddot{7}$ & 㞭 & 疍 & 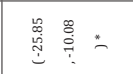 & $\stackrel{q}{\stackrel{p}{+}}$ & 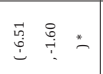 & 品 & 串: & 蒿 & 急荤 & $\approx$ & 一卷善 \\
\hline 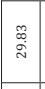 & 总总 & 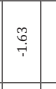 & 害要 & 苦 & 高 & 号 & 容営 & 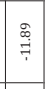 & 㟢育 & 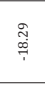 & 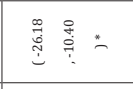 & 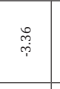 & 高 & & 善总 & 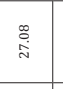 & 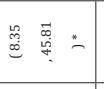 & $\dddot{\dddot{m}}$ & 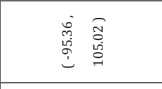 \\
\hline 总 & 垔善 & $\stackrel{8}{\rightarrow}$ & 咅 & 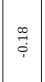 & 衰票 & $\stackrel{\sim}{\sim}$ & 悹覂 & 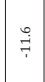 & 高离 & 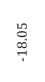 & 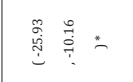 & $\overrightarrow{\tilde{\gamma}}$ & 高写 & 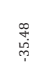 & 赏总: & $\stackrel{p}{\vec{N}}$ & 穿富 & $\vec{n}$ & 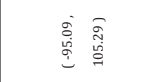 \\
\hline 蒡 & 总总二 & צֶ. & 要 & 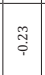 & 誉草: & 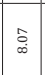 & 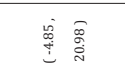 & $\stackrel{9}{\exists}$ & 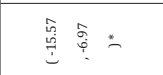 & $\frac{7}{7}$ & 㺃学: & $\vec{~}$ & 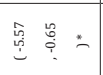 & & 䪐 & 萹 & 鲨言: & 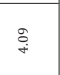 & 一亳总 \\
\hline 㠃 & 室高 & $\frac{\pi}{7}$ & 裹要 & : & 善夆 & 点 & 商璦 & 产 & 旁高 & 瓷 & 商寄 & 萨 & 高产 & 守 & 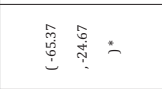 & 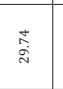 & 总裹 & : & 高要 \\
\hline 觠 & 蒿菜二 & 咢 & 要五 & $\frac{m}{a}$ & 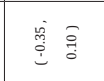 & $\stackrel{0}{\circ}$ & 悹愛 & 号 & 掌高 & 号 & 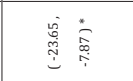 & $\overline{4}$ & 䔂要 & & 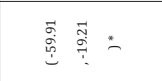 & 离 & 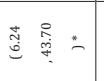 & 草 & 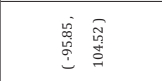 \\
\hline 嘉 & 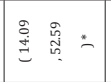 & 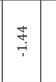 & 要 & $\frac{?}{3}$ & 裹営 & : & 高 & 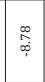 & 产娈 & $\approx$ & 密言 & $\%$ & 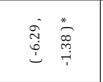 & & 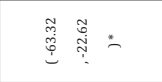 & 丞 & 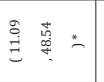 & $\stackrel{\sim}{\infty}$ & 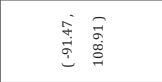 \\
\hline 㞼 & 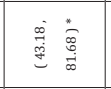 & $\vec{T}$ & 害票 & $\stackrel{n}{q}$ & 庇 & $\vec{\sigma}$ & 害总 & 亭 & 量方 & 离 & 善蒚 & : & 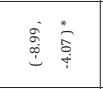 & & 畄苦 & 舘 & 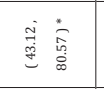 & 葶 & 奋要 \\
\hline 范 & 㿥蓠 & 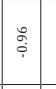 & 容总 & 品 & 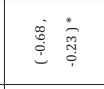 & हैं & 啇 & $\vec{\partial}$ & 荬 & 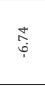 & 兽焉 & जि & ) & & 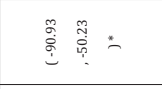 & 갱 & 高咅 & 号 & $\begin{array}{ll}0.5 \\
0\end{array}$ \\
\hline$\vec{i}$ & 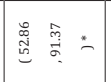 & 岂 & 宭票 & $\stackrel{\circ}{a}$ & 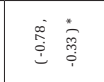 & 冞 & 高高 & $\stackrel{q}{\longrightarrow}$ & 突要 & F & 噢言 & 管 & 高高 & & 学学: & $\cong$ & 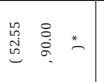 & 幕 & 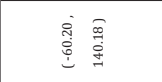 \\
\hline تัّ & 焘嵩 & $\stackrel{\square}{\square}$ & 要 & 畜 & 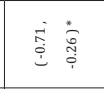 & $\frac{4}{4}$ & 商高 & 荌 & 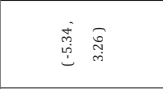 & $\frac{p}{p h}$ & 畄䙳 & 商 & 总 & & 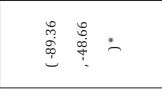 & : & 放 & 离 & 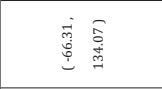 \\
\hline 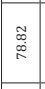 & 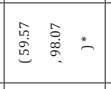 & 涼 & 这 & 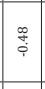 & 苔害 & 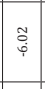 & 䓺要 & $\stackrel{m}{N}$ & 善番 & $\overrightarrow{\underline{n}}$ & 裹要 & 志 & 总总 & & 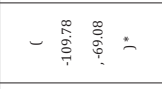 & 参 & 善哻二 & ì & 一咅金 \\
\hline 总 & 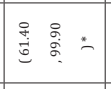 & $:$ & 窇䙳 & 号 & 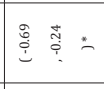 & 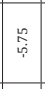 & 善 & 总 & 高埕 & $\stackrel{8}{8}$ & 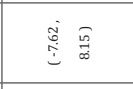 & \begin{tabular}{l}
\multirow{6}{*}{} \\
\end{tabular} & 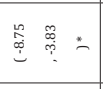 & & 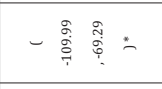 & 产 & 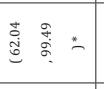 & ฐ & 一总拿 \\
\hline F & 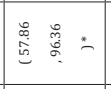 & $\stackrel{+}{t}$ & 总鬲 & : & 旁 & $\vec{b}$ & 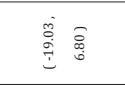 & $\because$ & 高高 & : & 高要 & 茫 & 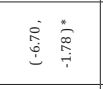 & & 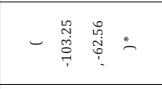 & $\stackrel{2}{E}$ & 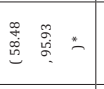 & \& & 量 \\
\hline 善 & 害喜 & 客 & 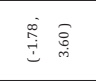 & 寄 & 單骂 & 点 & 总畄 & : & 荨高 & $\vec{\Xi}$ & 害总 & 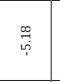 & 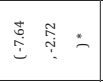 & & 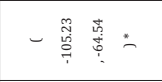 & 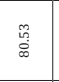 & 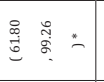 & $\stackrel{\approx}{\tilde{n}}$ & 一窬票 \\
\hline 品 & 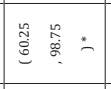 & $\vec{g}$ & 荨 & 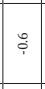 & 总粱 & 文 & 要 & जि & 产高 & : & 高要 & 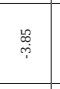 & 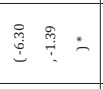 & & 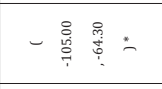 & 员 & 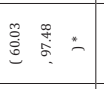 & 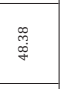 & 一总融离 \\
\hline$\approx$ & 歚善: 二 & 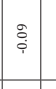 & 趂 & 客 & 总言 & $\approx$ & 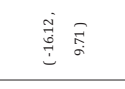 & \% & 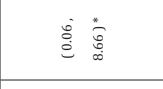 & $\stackrel{8}{\circ}$ & 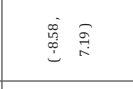 & $\overrightarrow{4}$ & 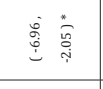 & & 一 器 & 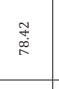 & 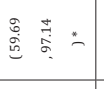 & 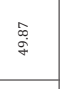 & 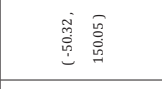 \\
\hline 蒪 & 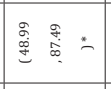 & : & 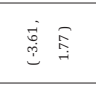 & 号 & 葛 & 号 & 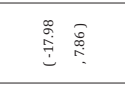 & 獣 & 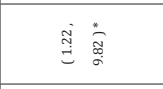 & $\stackrel{\text { m }}{\text { i }}$ & 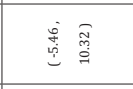 & 苧 & 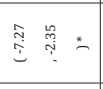 & & 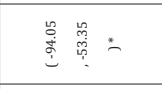 & 器 & 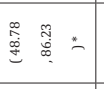 & 愛 & 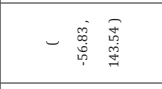 \\
\hline 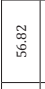 & 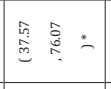 & 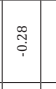 & 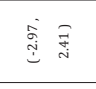 & 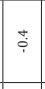 & 产 & 品 & 离 & $\cong$ & 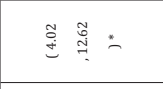 & $\stackrel{\%}{\varpi}$ & 或蒡: & $\frac{p}{q}$ & 釆嵩 & & 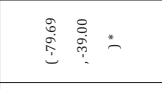 & 器 & 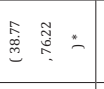 & $\stackrel{乛}{*}$ & 㶓 \\
\hline $\overrightarrow{\mathrm{g}}$ & 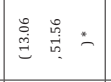 & 品 & 跑 & 旁 & 商言 & 密 & 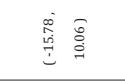 & 㩊 & 豆䒿: & $\stackrel{\circ}{\circ}$ & 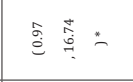 & $\stackrel{+}{+}$ & 实 & & 耪总 & స్ & 总泀 & $\stackrel{\overbrace{}}{\doteq}$ & 意畐 \\
\hline : & 䯩稆 & 号 & 眔 & 莣 & 产 & 畄 & 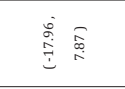 & o & 商融 & $\%$ & 离要 & $\tilde{y}$ & 总舜 & & 窎高 & 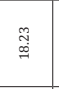 & 善点 & 声 & 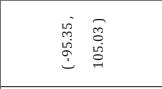 \\
\hline 莣 & 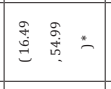 & \begin{tabular}{|c|}
2 \\
\end{tabular} & 営言 & 足 & 粟荌: & 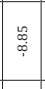 & 拿 & $\stackrel{q}{q}$ & 容察 & : & 商蛋 & \% & 总蓄二 & & 总虽虽 & 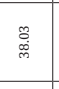 & 善总 & $\stackrel{\leftrightarrow}{\stackrel{2}{*}}$ & 藏尌一 \\
\hline 产 & 总 & 产 & 总 & 譬 & 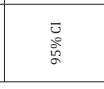 & 或 & 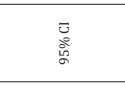 & & 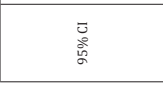 & & 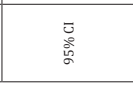 & 誉 & 产 & & 产 & 高 & 产 & 产 & 商 \\
\hline & $\frac{\bar{े}}{\mathrm{v}}$ & & 愛 & & 㐔 & & 흥 & & ⿳亠二口犬 & & 亏ัे & & )े & & 흠 & & 亏े & & - \\
\hline & 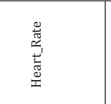 & & 高高喜 & & 高 & & 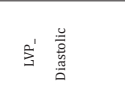 & & 高 & & 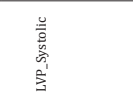 & & 言 & & 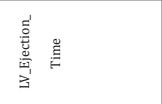 & & 㩊 & & 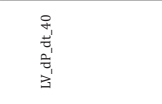 \\
\hline
\end{tabular}




\begin{tabular}{|c|c|c|c|c|c|c|c|c|c|c|c|c|c|c|c|}
\hline 喜 & 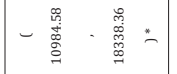 & 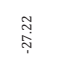 & 童高 & $\stackrel{\varrho}{\exists}$ & 产害 & $\frac{\tilde{y}}{\partial}$ & 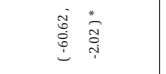 & Fे & 高嵒 & $\stackrel{n}{H}$ & 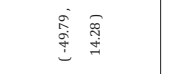 & & & & \\
\hline 言 & 高喜 & 产 & 一营葛: & $\stackrel{\leftrightarrow}{+}$ & 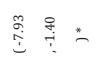 & $\stackrel{g}{q}$ & 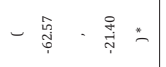 & 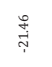 & 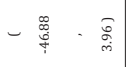 & 员 & 一号 & $\stackrel{乛}{\square}$ & 一尌票 & $\frac{\infty}{\alpha}$ & $-\stackrel{5}{0}$ \\
\hline 高 & 一总总总点 & 愛 & 密 & $\stackrel{\circ}{\circ}$ & 产突 & $\stackrel{\mathscr{p}}{\not ึ}$ & 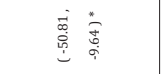 & $\stackrel{\text { F }}{\mathcal{F}}$ & 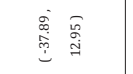 & $\stackrel{\circ}{\%}$ & 衰畐 & 突 & 鬲否 & $\stackrel{Ð}{\square}$ & 商 \\
\hline 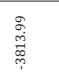 & 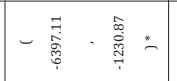 & : & 产咅 & 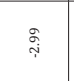 & 彥 & 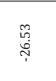 & 言害 & $\underset{\Phi^{*}}{\sim}$ & 高 & $\stackrel{\circ}{\overparen{H}}$ & 害蛋 & 愛 & 咅高 & 商 & 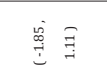 \\
\hline 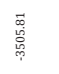 & 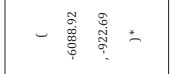 & $F$ & 美要 & ซิ & 虽哭: & 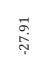 & 丞管 & $\stackrel{\circ}{\%}$ & 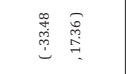 & $\stackrel{\mathscr{m}}{\underset{7}{7}}$ & 善票 & $\stackrel{\circ}{\circ}$ & ํํㄹ & 望 & 寻宫 \\
\hline 㩊 & 一誉愛: & 品 & 突 & ఫ̊ & 㤐要 & 約 & 熟庐 & $\stackrel{8}{\stackrel{q}{q}}$ & 商 & 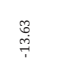 & 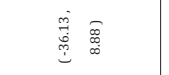 & $\stackrel{\circ}{\leftrightarrow:}$ & 要 & $\stackrel{\infty}{g}$ & 宷 \\
\hline 蔃 & 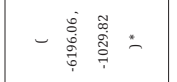 & $\overrightarrow{v_{0}}$ & 总 & \%ั] & ㅇํำ & ఃั & 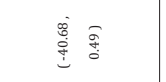 & 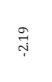 & 高覂 & 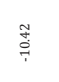 & 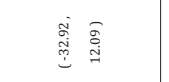 & 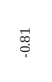 & 窎 & $\stackrel{\%}{\%}$ & 亭㩊 \\
\hline 鲖 & 喜营: & 产 & 一 荨票 & $\stackrel{3}{3}$ & 衰䍃 & 号 & 总总离 & 总 & 一毫需 & 高 & 唯素: & $\stackrel{\sharp}{\rightrightarrows}$ & 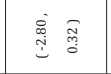 & $F$ & 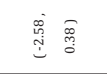 \\
\hline 苋 & 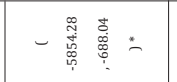 & :े̆ & 尌䍃 & $\stackrel{\infty}{\longrightarrow}$ & 善要 & 苟 & 费总 & $\stackrel{\vec{g}}{\stackrel{9}{9}}$ & 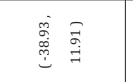 & 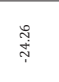 & 高旁 & $\stackrel{0}{9}$ & ปู่ & 品 & 姜槅 \\
\hline 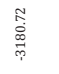 & 一嘉惹 & 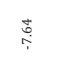 & 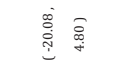 & $\stackrel{\vec{g}}{\longrightarrow}$ & 害孚 & 㵙 & 总亭 & $\stackrel{9}{7}$ & 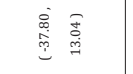 & $\stackrel{?}{乛}$ & 善豆 & $\stackrel{\vec{u}}{\vec{p}}$ & 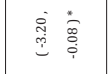 & 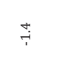 & 受 \\
\hline 商 & 一逽总: & के & 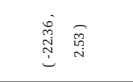 & $\stackrel{n}{g}$ & 覃 & \%ัّ & 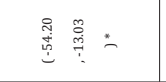 & : & 㩊哥 & 产 & 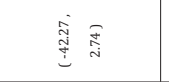 & $\dddot{m}$ & 产 & $\stackrel{\leftrightarrow}{\longrightarrow}$ & 耐愛 \\
\hline 㯊 & 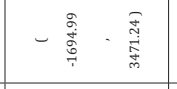 & $\stackrel{\overrightarrow{\underline{v}}}{\vec{y}}$ & 芴高 & $\stackrel{8}{\dddot{8}}$ & 高营 & $\stackrel{n}{:}$ & 总㻤 & 鰥 & 璦育 & 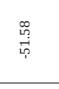 & 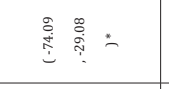 & $\stackrel{\square}{\square}$ & 旁琴 & $\stackrel{\infty}{\square}$ & ชู่ \\
\hline ఫ్ّ & 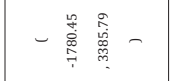 & 獣 & 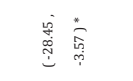 & 爱 & 畹商 & : & 总总离: & $\stackrel{\mathscr{g}}{\%}$ & 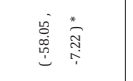 & ఖั. & 虽旁: & $\stackrel{\infty}{\square}$ & 高 & $\stackrel{\circ}{\square}$ & 高 \\
\hline 总 & 一 & $\frac{7}{i}$ & 商育 & $\vec{b}$ & 商离 & \% & 总毒: & $\stackrel{q}{q}$ & 告 & 商 & 虽虽 & $\stackrel{\approx}{\dddot{z}}$ & 㩊 & : & 豈高 \\
\hline 醞 & 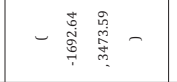 & : & 离高 & \% & 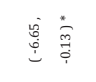 & 总 & 善骂 & $\stackrel{\infty}{\vec{p}}$ & 言畺 & $\underset{\substack{0 \\
\tilde{n}}}{ }$ & 总蒿 & $\stackrel{\frac{p}{g}}{\square}$ & 害票 & $\stackrel{+}{a}$ & 兽 \\
\hline 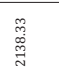 & 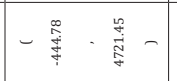 & 㩊 & 啹憘: & 虽 & 萬 & $\frac{7}{9}$ & - & . & 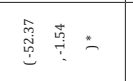 & 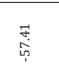 & 憘总 & 器 & 咅愛 & : & 产 \\
\hline 気 & 一喜。営一 & $\stackrel{\mathrm{g}}{\mathrm{g}}$ & 产总: & $\stackrel{+}{+}$ & 童害: & : & 一嚍素之 & $\frac{\tilde{g}}{\mathrm{~g}}$ & 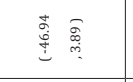 & 㩊 & 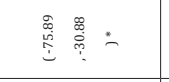 & $\underline{n}$ & 言 & $\underline{9}$ & 高 \\
\hline 产 & — 产蒿 & 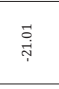 & 窖底 & 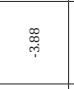 & 产 & 㩊 & 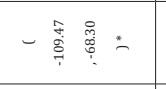 & 举 & 罣票 & 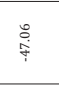 & 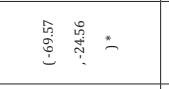 & 骂 & 总 & 药 & 高愛 \\
\hline 㩊 & 一辡䓂 & 谷 & 啹 & $\frac{1}{4}$ & 高融 & 䐱 & 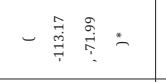 & 产 & 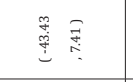 & 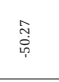 & ํํำ & \% & 孚票 & 영 & 总预 \\
\hline 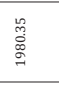 & 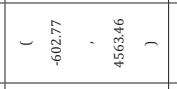 & 茄 & 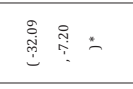 & 票 & 葛草: & 号 & 一 $\stackrel{n}{\exists}$ & $\overrightarrow{\tilde{z}}$ & 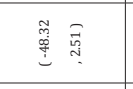 & 蒂 & 帝言帝二 & 范 & 尊畐 & $\stackrel{0}{\circ}$ & 尊票 \\
\hline 善 & 一离拿 & 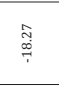 & 咅商 & . & 苞产 & 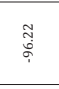 & 一总总总: & $\overrightarrow{\underline{0}}$ & 害高 & $\frac{⿱}{9}$ & 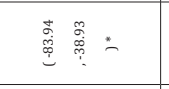 & 䓂 & 产 & $\stackrel{m}{:}$ & 愛 \\
\hline 总 & 一盖 & $\stackrel{\vec{\sigma}}{\mathrm{F}}$ & 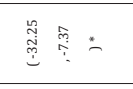 & $\dot{4}$ & 害高 & 営 & 一镐管: & 管 & 管: & 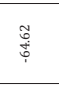 & 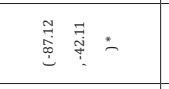 & 品 & 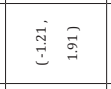 & : & 我愛 \\
\hline 畄 & 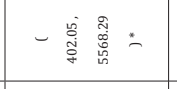 & $\underset{\ddot{m}}{\underline{m}}$ & 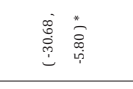 & v & 芍 & 薈 & 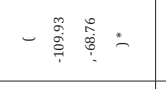 & 产 & 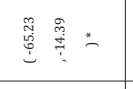 & $\frac{\infty}{\square}$ & 愛离 & $\stackrel{\circ}{\circ}$ & 离 & 菅 & 可 \\
\hline$\underset{\mathscr{W}}{\mathscr{W}}$ & 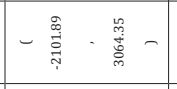 & Ф. & 要要 & $\stackrel{\circ}{\longrightarrow}$ & 害垔 & 总 & 喜虽 & $\frac{\tilde{z}}{\tilde{w}}$ & 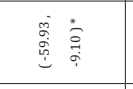 & $\stackrel{n}{4}$ & 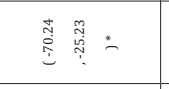 & 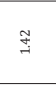 & 产 & $\Xi$ & 商 \\
\hline 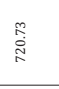 & 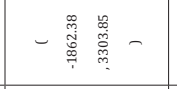 & $\stackrel{\mathscr{P}}{*}$ & 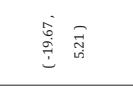 & 高 & 栾勇 & $\stackrel{\mathscr{\varpi}}{\mathscr{\Psi}}$ & 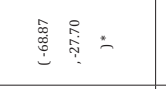 & : & 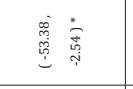 & $\overrightarrow{\mathrm{g}}$ & 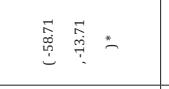 & 号 & ปี่ & 商 & 守寄 \\
\hline 兽 & 一巻 & 范 & 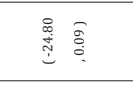 & 㞼 & 高票 & 烹 & 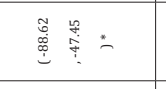 & 产 & 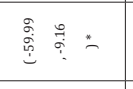 & 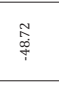 & 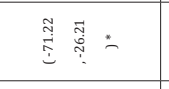 & $\frac{5}{\partial}$ & 赵 & : & 㦼孚 \\
\hline$\frac{5}{2}$ & 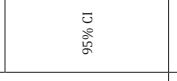 & $\frac{\sqrt{2}}{2}$ & 善 & 高 & 愛 & $\frac{\sqrt{5}}{2}$ & 商 & $\frac{\sqrt{5}}{2}$ & 总 & $\frac{\sqrt{5}}{2}$ & 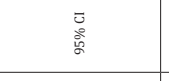 & $\frac{\sqrt{3}}{2}$ & 关 & $\frac{1}{2}$ & 总 \\
\hline & 亏े̀ & 善 & & 言 & & & $\frac{\bar{g}}{\mathrm{v}}$ & & 蒿 & & $\overrightarrow{\mathrm{g}}$ & & 器 & & 总兽 \\
\hline & & 熟 & & 岁咅 & & & & & 憘 & & 哭愛 & & 竞 & & $\begin{array}{l}\frac{1}{2} \\
\frac{1}{2} \\
\frac{1}{2}\end{array}$ \\
\hline
\end{tabular}




\begin{tabular}{|c|c|c|c|c|c|c|c|c|c|c|c|c|c|c|c|}
\hline & & & & & & & & & & & & & & $\vec{I}$ & 言票 \\
\hline 葶 & 驾 & $\underset{⿱ 亠 巾}{i}$ & 一害畐 & $\stackrel{m}{i}$ & 一㝓 & ț & 一高票 & : & 一离鬲 & 樀 & 一菤 一融 & 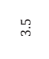 & - 嵩嵩 & $\stackrel{9}{+}$ & 롱 \\
\hline : & 誉 & 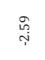 & 高㔄 & : & 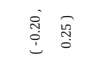 & ঙุ & 咅孚 & 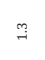 & 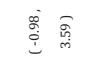 & : ì & 言弯 & $\stackrel{p}{=}$ & 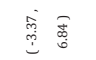 & 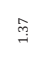 & 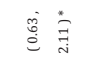 \\
\hline$\stackrel{艹}{\dot{t}}$ & 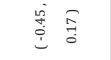 & $\stackrel{\Re}{\dddot{T}}$ & 总榢 & 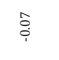 & 商愛 & $\stackrel{\leftrightarrow}{\leftrightarrow}$ & 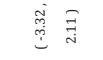 & 菅 & 苞 & 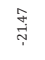 & 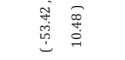 & $\stackrel{+}{\circ}$ & 总焉 & $\$$ & 突言 \\
\hline 草 & 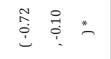 & 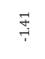 & 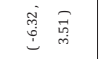 & $\stackrel{\circ}{a}$ & 衰票 & 寞 & 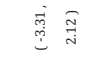 & gू & 咅鬲 & 总 & 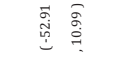 & 学 & 芭芭 & $\stackrel{q}{+}$ & 唐 \\
\hline 훔 & 芳 & $\stackrel{9}{9}$ & 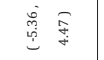 & $\stackrel{\text { g }}{i}$ & 高要 & : & 总 & $\frac{\circ}{3}$ & 羿䓓 & 足 & 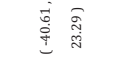 & $\mathscr{\infty}_{\infty}^{\infty}$ & 密 & $\cong$ & 営蓠 \\
\hline 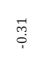 & 夤 & ָू & 总踓 & $\stackrel{m}{3}$ & 鳏高 & $\stackrel{9}{\forall}$ & 害踓 & $\stackrel{9}{I}$ & 密塄 & 㩊 & 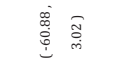 & 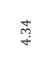 & 酉重 & $\stackrel{\infty}{\exists}$ & 善离 \\
\hline ⿳艹冖్⿱口巾: & 骂 & gू & 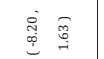 & $\stackrel{8}{9}$ & 商 & $\stackrel{8}{\longrightarrow}$ & 窝 & 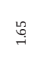 & 旁商 & 哭 & 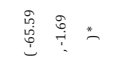 & 㴽 & 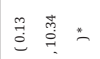 & 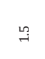 & 号莡 \\
\hline$\stackrel{\vec{A}}{i}$ & 总酻 & के & 离 & \%ั & 愛 & $\stackrel{\infty}{\rightarrow+}$ & 裹手 & $\stackrel{\vec{I}}{I}$ & 衰 & 总 & 言言 & 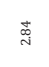 & 突 & 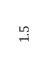 & 突 \\
\hline ֻั & 商愛 & 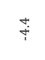 & 旁鬲 & $\stackrel{\square}{\vec{u}}$ & 总 & הู & 案亳 & $\stackrel{\text { N }}{*}$ & 产 & $\stackrel{\infty}{\vec{q}}$ & 商商 & $\dddot{\Re}$ & 离 & 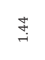 & 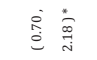 \\
\hline 受 & 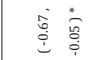 & \%్ & 厗票 & $\stackrel{\circ}{\frac{\partial}{i}}$ & 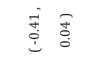 & $\stackrel{\mathscr{q}}{7}$ & 享要 & 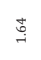 & 釆票 & 睪 & 孚 & $\vec{n}$ & 果宫 & $\stackrel{\circ}{\rightarrow}$ & 突害 \\
\hline$\stackrel{\mathscr{O}}{9}$ & 焉窟 & $\dddot{Y}$ & 蔩融 & 畹 & 离嵩 & $\frac{8}{8}$ & 高愛 & 菅 & 峖 & $\underset{\Phi}{*}$ & 害高 & $\stackrel{\circ}{\infty}$ & 总 & 苛 & 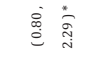 \\
\hline कू & 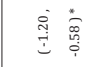 & o̊ & 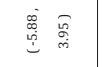 & 旁 & 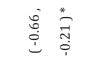 & $\frac{9}{3}$ & 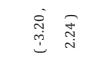 & $\frac{9}{\partial}$ & 啇 & : & 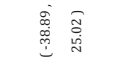 & : & 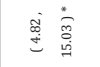 & 㕝 & 高宭 \\
\hline 总 & 高蹗 & $\dddot{\varpi}$ & 商票 & $\stackrel{\frac{m}{i}}{i}$ & 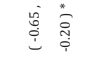 & 今 & ชู่ & 总 & 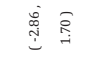 & 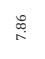 & 高高 & $\stackrel{g}{g}$ & 菤总 & $\ddot{g}$ & 摘 \\
\hline 愛 & 美寄 & $\frac{\%}{\partial}$ & 言酻 & 厗 & 高㫄 & 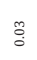 & 高 & $\overrightarrow{7}$ & 离高 & 吊 & 总高 & $\underset{\infty}{\tilde{\infty}}$ & 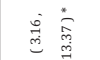 & $\stackrel{\approx}{T}$ & 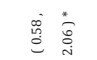 \\
\hline 总 & 蒮葶: & 品 & 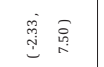 & $\stackrel{4}{*}$ & - & $\vec{g}$ & $\vec{\exists} \bar{\exists} \bar{\Xi}$ & بَ & 㩊 & 薆 & 总 & $\stackrel{\circ}{\infty}$ & 品哭 & $\cong$ & 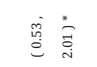 \\
\hline s. & 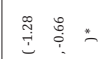 & \% & 羑畨二 & $\overrightarrow{\vec{r}}$ & 管 & 学 & 全 & 㞼 & 草㔛: & 兽 & 器卷: & $\vec{\Phi}$ & 总楚 & $\stackrel{g}{\square}$ & 器咅 \\
\hline 葶 & 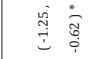 & ले & 岁 & $\stackrel{7}{i}$ & 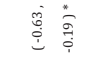 & $\frac{8}{N}$ & 总蛋 & $\stackrel{\square}{\longrightarrow}$ & 窎 & 睪 & 害㞼 & 总 & శ్ర్తి & 害 & 啇 \\
\hline 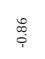 & 姜营: & ח & ชู่ & 骂 & 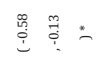 & $\stackrel{g}{g}$ & 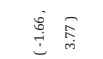 & $\stackrel{\text { q̆ }}{\longrightarrow}$ & 鹃票 & : & 急 & $\stackrel{\mathscr{N}}{\sim}$ & - & ळ & 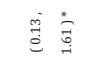 \\
\hline$\stackrel{9}{\dddot{\%}}$ & 䓵管 & $\stackrel{i}{\stackrel{i}{*}}$ & 窇 & $\stackrel{*}{\vdots}$ & ำ & $\frac{g}{\partial}$ & 달 & 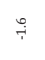 & 菢嵒 & 点 & 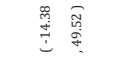 & $\stackrel{\infty}{=}$ & 虽总 & $\stackrel{\circ}{\circ}$ & 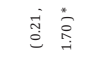 \\
\hline 7 & 辛承 & $\cong$ & 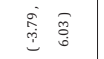 & : & 容高 & 喜 & 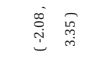 & 帝 & 高 & $\sigma$ & 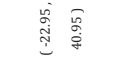 & : & 骂总 & $g$ & 葬离 \\
\hline 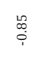 & 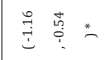 & 莒 & 於㩊 & $\stackrel{7}{\vec{z}}$ & - & 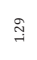 & 害䙳 & $\stackrel{m}{q}$ & 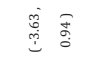 & 兽 & 总愛 & $\vec{\sigma}$ & 总䙵二 & $\cong$ & 器 \\
\hline : & 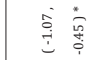 & 哭 & 害蛋 & 曾 & 总宰 & $\stackrel{q}{\sharp}$ & 害 & $\stackrel{\pi}{\overrightarrow{7}}$ & 衰踓 & 总 & 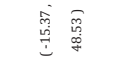 & สี & 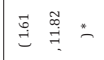 & $\stackrel{8}{\rightarrow}$ & 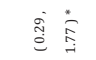 \\
\hline 薃 & 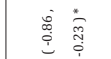 & 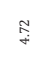 & 高害票 & ज्ञ & 急 & $\stackrel{m}{\mathrm{~N}}$ & 高票 & 总 & 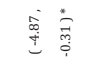 & 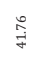 & 要营 & 爰 & 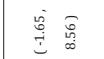 & $\stackrel{\%}{\circ}$ & 害离 \\
\hline 。ั & 高 & 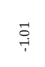 & 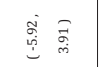 & 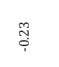 & 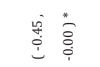 & 高 & 商 & : & 啇突 & $\stackrel{8}{\longrightarrow}$ & 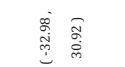 & $\stackrel{\circ}{\circ}$ & 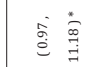 & ळ & 哭富 \\
\hline$\stackrel{\varpi}{\stackrel{\varpi}{i}}$ & 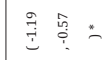 & $\stackrel{\circ}{\exists}$ & 容㩊 & $\stackrel{q}{a}$ & - & : & 产 & : & 害 & 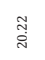 & - 一 & $\frac{\rho}{\infty}$ & 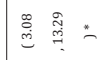 & : & 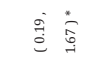 \\
\hline 㲋 & 愛 & $\frac{\bar{s}}{\frac{5}{2}}$ & 商 & 产 & 离 & 童 & 总 & 产 & 总 & 言 & 丞 & 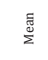 & 产 & 言 & 产 \\
\hline & 営 & & \multicolumn{2}{|c|}{ 㐔 } & \multicolumn{2}{|c|}{ 善 } & 营 & & 苛 & & \multicolumn{2}{|c|}{ ปे } & \multicolumn{2}{|c|}{$\bar{v}$} \\
\hline \multicolumn{2}{|c|}{ 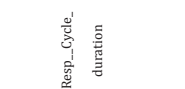 } & 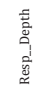 & & & \multicolumn{2}{|c|}{ 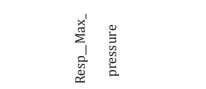 } & 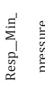 & & 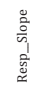 & & \multicolumn{2}{|c|}{$\frac{2}{2}$} & \multicolumn{2}{|c|}{ 言 } \\
\hline
\end{tabular}


The blood pressure group showed a statistically significant decrease in pressure, beginning at the third hour post challenge, which remained significant until completion of monitoring (Figure 1) and (Table 2). Aortic Mean Pressure (AMP) had dropped to a mean of $82.8 \mathrm{mmHg}$ by the third hour, and decreased to $72.4 \mathrm{mmHg}$ by the sixth hour post monitoring. The corresponding mean AMP from the pre-challenge data remained in the range of 90 to $95 \mathrm{mmHg}$ throughout the course of the study. The AMP was an average of 11.27 (95\% CI 1.00 to 21.55) and $20.06 \mathrm{mmHg}$ (95\% CI 9.79 to 30.33 ) below pre-challenge levels at 3 and 5 hours post-challenge, respectively.

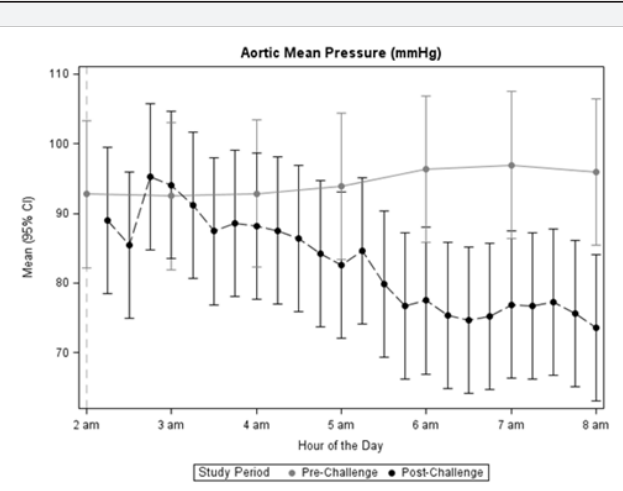

Figure 1: Changes in Aortic Mean Pressure (mmHG) over time after intravenous intoxication of SEB in rhesus macaques. Dotted, dark line represents data collected post-intoxication. Solid, gray line represents normal data collected from the same animals pre-intoxication as a control for diurnal variation. Data represents the mean from three rhesus macaques.

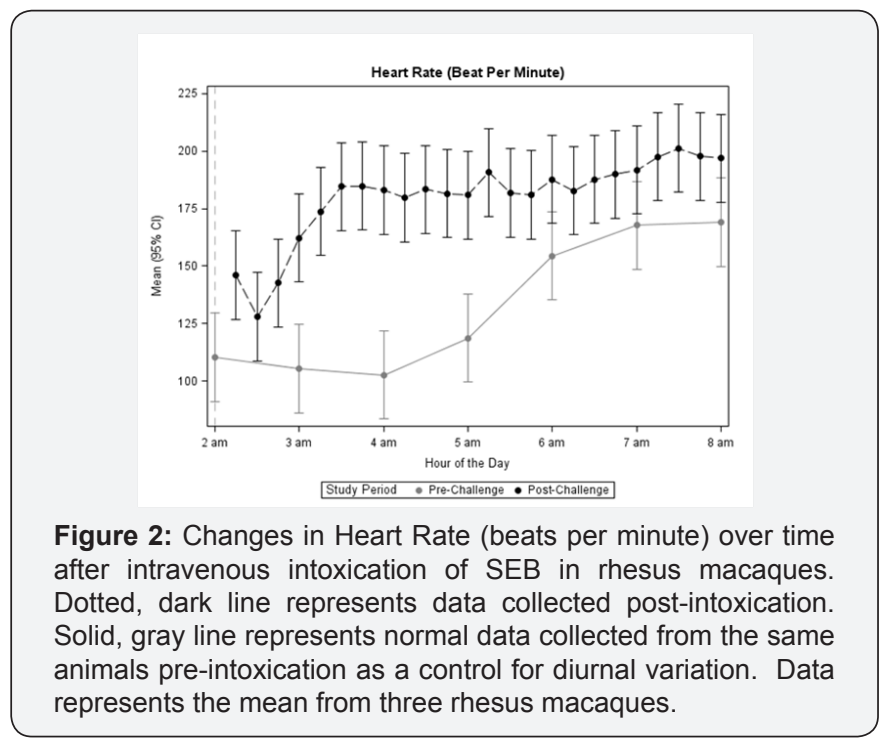

Average heart rate was significantly increased throughout the post challenge monitoring period (Figure 2) and (Table 2). It reached a low of $103.7 \mathrm{bpm}$ in first hour post challenge then rapidly increased to $180.8 \mathrm{bpm}$ during the second hour. It remained elevated in the $180-200 \mathrm{bpm}$ range for the remainder of the post challenge monitoring period. The maximum and minimum values of the corresponding pre-challenge data were 168.9 and $103.6 \mathrm{bpm}$, respectively. Heart rate was elevated above pre-challenge values by $80.4 \mathrm{bpm}(95 \% \mathrm{CI} 61.1$ to 99.6$)$ at 2 hours post challenge.

Table 2: A representative parameter from each of the five groups demonstrates the mean effect of challenge with SEB over time. An '*' indicates a significant change from pre-challenge after controlling for diurnal variations. Values indicate mean $(95 \% \mathrm{Cl})$.

\begin{tabular}{|c|c|c|c|c|c|}
\hline & \multicolumn{5}{|c|}{ Hours Post Challenge } \\
\cline { 2 - 6 } & $\mathbf{1}$ & $\mathbf{2}$ & $\mathbf{3}$ & $\mathbf{4}$ & $\mathbf{5}$ \\
\hline $\begin{array}{c}\text { Aortic Mean Pressure } \\
\text { (mmHg) }\end{array}$ & $1.63(-8.64,11.90)$ & $-4.65(-14.93,5.62)$ & $-11.27(-21.55,-1.00)^{*}$ & $-18.85(-29.12,-8.58)^{*}$ & $-20.06(-30.33,-9.79)^{*}$ \\
\hline $\begin{array}{c}\text { Heart Rate (Beat Per } \\
\text { Minute) }\end{array}$ & $56.82(37.57,76.07)^{*}$ & $80.36(61.11,99.61)^{*}$ & $62.24(42.99,81.49)^{*}$ & $33.34(14.09,52.59)^{*}$ & $24.09(4.84,43.34)^{*}$ \\
\hline $\begin{array}{c}\text { QTcFridericia (msec) } \\
\text { Respiration Rate } \\
\text { (Breath Per Minute) }\end{array}$ & $\begin{array}{c}-61.18(-83.68,- \\
38.68)^{*}\end{array}$ & $\begin{array}{c}-50.27(-72.78,- \\
27.77)^{*}\end{array}$ & $\begin{array}{c}-51.25(-73.75,- \\
28.74)^{*}\end{array}$ & $-19.76(-42.27,2.74)$ & $-10.42(-32.92$, \\
\hline $\begin{array}{c}\text { Temperature } \\
\text { (centigrade) }\end{array}$ & $1.03(0.29,1.77,11.82) *$ & $7.83(2.72,12.93)^{*}$ & $8.27(3.16,13.37)^{*}$ & $5.51(0.40,10.61)^{*}$ & $4.34(-0.77,9.44)$ \\
\hline
\end{tabular}

The ECG intervals were shortened throughout (Figure 3) and (Table 2). QTcFridericia, a corrected QT interval measurement used to detect cardiac arrhythmias, began at $343 \mathrm{msec}$, and continued to shorten to $278 \mathrm{msec}$ by the end of the monitoring period. It should be noted, however, that these values were only significantly different from pre-challenge control up to the fourth hour of monitoring post challenge. The mean interval from the pre-challenge data ranged from 375 to $310 \mathrm{msec}$. The difference from pre-challenge controls was greatest at 1 hour post challenge, where mean QTc duration was $61 \mathrm{msec}$ (95\% CI 39 to 84 ) below pre-challenge levels.
Mean respiratory rate was increased throughout, with statistical significance up to the fifth hour of monitoring (Figure 4) and (Table 2). The post challenge rates varied between 24.2 and $34.4 \mathrm{bpm}$, while the pre-challenge data showed a gradual increase from 20.5 to $27.0 \mathrm{bpm}$. Corresponding Expiratory Times and Respiration Cycles were decreased. No significant changes were noted in intrathoracic pressures, respiratory depth, or respiratory slope.

Temperature was significantly elevated throughout the post challenge monitoring period (Figure 5) and (Table 2). The first 
two hours post-challenge showed only slight variation with mean temperatures in the range of 38.0-38.4oC. Temperature then climbed sharply in the third and fourth hours to an average of 39.2oC. The peak measurement in the final hour of monitoring was 39.6oC. Pre-challenge data showed a gradual increase from 37.5-38.1oC. The difference from pre-challenge control increased to a maximum of $1.4 \mathrm{oC}(95 \%$ CI 0.7 to 2.1 ) by 4 hours post-exposure.

\section{Discussion}

SEB intoxication has been shown to cause a wide spectrum of symptoms, dependent on the route of exposure. The rhesus macaque has been used as a model for inhalational and intravascular SEB intoxication [15-25]. While initial results have been promising, particularly for the inhalational model, the data collected has focused on hematological values, tissue pathology, and clinical signs observed. The physiological data has largely been limited to body temperature. Radiotelemetry can provide a wealth of physiological information that is currently lacking from the literature. This data has the potential to further elucidate the pathophysiological development of the disease state in these models and may further validate them as an accurate representation of the human disease syndromes.

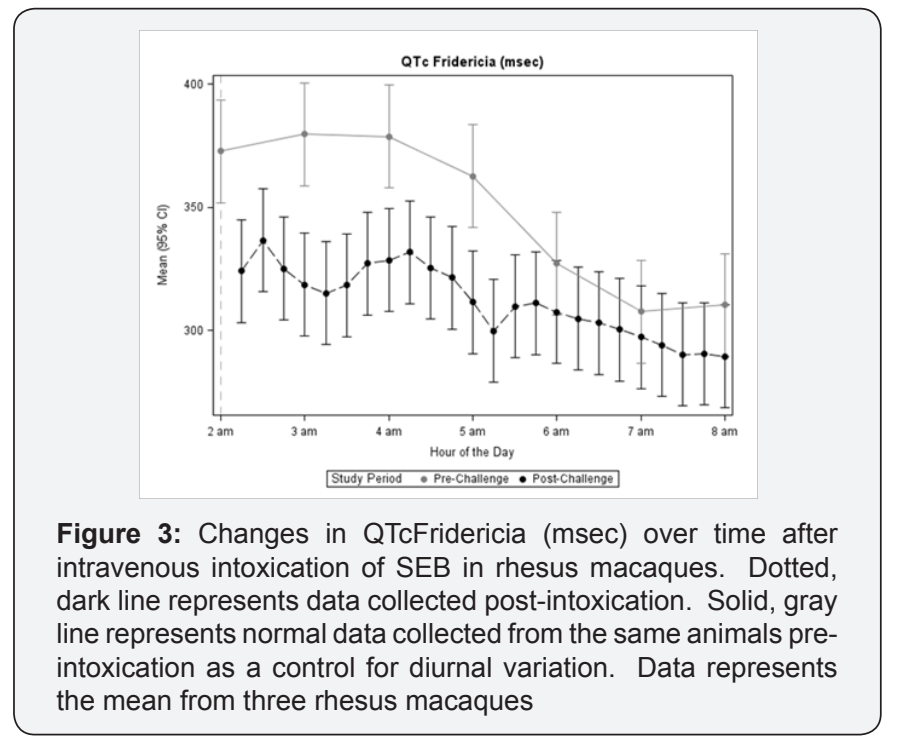

The first objective of this study was to establish a baseline of physiological parameters for the rhesus macaque, using radiotelemetry, after intravenous injection of SEB. Even with the limited numbers of animals used, there were several significant trends noted. Aortic Mean Pressure dropped significantly during the third hour and never recovered (Figure 1) and (Table 2). Heart Rate rose dramatically in the first two hours, then tapered off to a minor increase in the final three (Figure 2) and (Table 2). ECG intervals were all shortened, more significantly so during the first three hours of monitoring (Figure 3) and (Table 2). Respiration Rate and Temperature gradually increased throughout, with the exception of a short but steep temperature increase in the third hour (Figures 4 \& 5) and (Table 2).
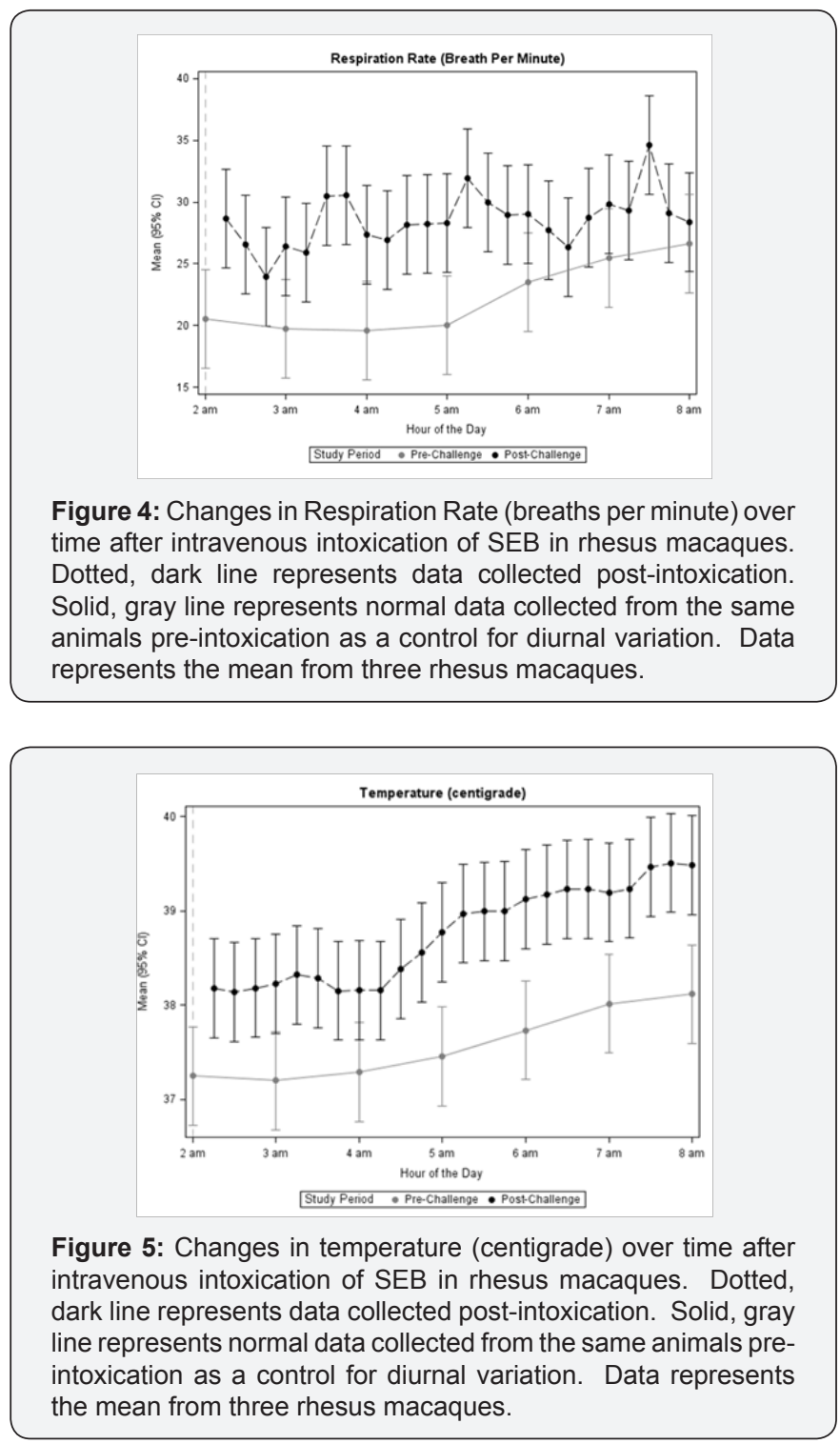

The second objective was to determine the validity of the rhesus macaque as a model for human SEB intoxication. The rapid onset of physiologic effects after intravenous SEB intoxication, as observed by telemetry in rhesus macaques, directly correlates to the clinical picture of human intoxication. The gastrointestinal, mucocutaneous, and inhalational routes of exposure in humans all demonstrated an onset of clinical signs within minutes to hours of exposure $[1-4,9,10]$. The specific findings of decreased Aortic Mean Pressure, increased Heart Rate, and increased Temperature found in the macaques are a good representation of the clinical features of human patients suffering from a systemic intoxication.

\section{Conclusion}

The complex physiological data recorded via the T27F1Bradiotelemetry device represents the most complete picture of the systemic intoxication syndrome in the literature, and may be used as a baseline for future studies involving SEB intoxication 
in nonhuman primates. The findings from this study support the use of the rhesus macaque as a model for systemic intoxication. This study observed three rhesus macaques for a period of five hours after intravenous intoxication with SEB. To further elucidate the pathophysiology of SEB, future studies may include different routes of exposure, increased periods of observation, increased numbers of animals, and different concentrations of the toxin.

\section{Acknowledgement}

The authors thank the Joint Science and Technology Office for Chemical and Biological Defense (JSTO-CBD) Defense Threat Reduction Agency (DTRA) for funding this research project (Project number CB-3680). The authors appreciate and recognize the expertise of the animal care staff of USAMRIID for their technical assistance and support throughout the duration of this project. Opinions, interpretations, conclusions, and recommendations are those of the author and are not necessarily endorsed by the U.S. Army.

\section{References}

1. Pinchuk IV, Beswick EJ, Reyes VE (2010) Staphylococcalenterotoxins. Toxins (Basel) 2(8): 2177-2197.

2. Fries BC, Varshney AK (2013) Bacterial Toxins-Staphylococcal Enterotoxin B. Microbiology Spectru 1(2).

3. Ortega E, Abriouel H, Lucas R, Galvez A (2010) Multiple roles of Staphylococcus aureus enterotoxins: pathogenicity, superantigenic activity, and correlation to antibiotic resistance. Toxins (Basel) 2(8): 2117-2131.

4. Ulrich RG, Wilhelmsen CL, Krakauer TD (2007) Staphylococcal Enterotoxin B and related toxins. ZF. Dembek, Textbook of Military Medicine. Office of the Surgeon General, Borden Institute, Washington DC, USA, pp. 311-22.

5. Scallan E, Hoekstra RM, Angulo FJ, Tauxe RV, Widdowson MA, et al. (2011) Foodborne illness acquired in the United States-major pathogens. Emerg Infect Dis 17:7-15.

6. The European Union Summary Report on Trends and Sources of Zoonoses, Zoonotic Agents and food-borne outbreaks in 2009. EFSA J 2011: 2090 .

7. DeVries Aaron S, Lindsey Lesher, Patrick M. Schlievert, Tyson Rogers, Lourdes G. Villaume et al. (2011) Staphylococcal Toxic Shock Syndrome 2000-2006: Epidemiology, Clinical Features, and Molecular Characteristics. Ed BinhAn Diep PLoS ONE 6(8): e22997.

8. Korch G, Lindle LE, Lebeda FJ (2005) Biological weapons defense: infectious diseases and counterbioterrorism. Totowa, Humana Press, New Jersey, USA.

9. Bergdoll MS (1983) Enterotoxins In Easmon CSF, Adlam C (eds.), Staphylococci and Staphylococcal Infections. Academic Press, London, pp. 559.

10. Rusnak JM, Kortepeter M, Ulrich R, Poli M, Boudreau E (2004) Laboratory exposures to staphylococcal enterotoxin B. Emerg Infect Dis 10: $1544-1549$.

11. Lee VT, Chang AH, Chow AW (1992) Detection of staphylococcal enterotoxin B among toxic shock syndrome (TSS)- and nonTSSassociatedStaphylococcus aureus isolates. J Infect Dis 166: 911915.

12. Kashiwada T, Kikuchi K, Abe S, Kato H, Hayashi H, Morimoto T, et al
(2012) Staphylococcal enterotoxin B toxic shock syndrome induced by community-acquired methicillin-resistant Staphylococcus aureus (CAMRSA). Intern Med 51(21): 3085-3088.

13. Wharton M, Chorba TL, Vogt RL (1990) Case definitions for public health surveillance. MMWR Recomm Rep 39: 1-50.

14. Andrews MM, Parent EM, Barry M, Parsonnet J (2001) Recurrent nonmenstrual toxic shock syndrome: clinical manifestations, diagnosis, and treatment. Clin Infect Dis 32: 1470-1479.

15. Weng C-F, Komisar JL, Hunt RE, Johnson AJ, Pitt ML, et al. (1997) Immediate responses of leukocytes, cytokines and glucocorticoid hormones in blood circulation of monkeys following challenge with aerosolized staphylococcal enterotoxin B. Internat.Immunol 9(12): 1825-1836.

16. Boles JW, Pitt ML, LeClaire RD, Gibbs P, Torres E, et al. (2003) Generation of protective immunity by inactivated recombinant staphylococcal enterotoxin $\mathrm{B}$ vaccine in nonhuman primates and identification of correlates of immunity. Clinical Immunology 108: 51-59.

17. Boles JW, Pitt ML, LeClaire RD, Gibbs PH, Ulrich RG, et al. (2003) Correlation of body temperature with protection against staphylococcal enterotoxin B exposure and use in determining vaccine dose-schedule. Vaccine 21: 2791-2796.

18. Lowell GH, Colleton C, Frost D, Kaminski RW, Hughes M, et al. (1996) Immunogenicity and efficacy against lethal aerosol staphylococcal enterotoxin B challenge in monkeys by intramuscular and respiratory delivery of proteosome-toxoid vaccines. Infection and Immunity 64: 4686-4693.

19. Tseng J, Komisar JL, Trout RN, Hunt RE, Chen JY (1995) Humoral immunity to aerosolized staphylococcal enterotoxin B (SEB), a superantigen, in monkeys vaccinated with SEB toxoid-containing microspheres. Infection and Immunity 63: 2880-2885.

20. Tseng J, Komisar JL, Chen JY, Hunt RE, Johnson AJ, et al. (1993) Immunity and responses of circulating leukocytes and lymphocytes in monkeys to aerosolized staphylococcal enterotoxin B. Infection and Immunity 61: 391-398.

21. Komisar JL, Weng C, Oyejide A, Hunt RE, Briscoe C, et al. (2001) Cellular and Cytokine Responses in the Circulation and Tissue Reactions in the Lung of Rhesus Monkeys (Macacamulatta) Pretreated with Cyclosporin A and Challenged with Staphylococcal Enterotoxin B. Toxicologic Pathology 29(3): 369-378.

22. Hodoval LF, Morris EL, Crawley GJ, Beisel WR (1968) Pathogenesis of Lethal Shock After Intravenous Staphylococcal Enterotoxin B in Monkeys. Applied Microbiology 16(2): 187-192.

23. Mattix ME, RE Hunt, CL Wilhelmsen, AJ Johnson, W Baze (1995) Aerosolized staphylococcal enterotoxin B-induced pulmonary lesions in rhesus monkeys (Macacamulatta). Toxicol Pathol 23: 262-268.

24. Soto PJ, Roessler WG (1965) Staphylococcal enterotoxemia: Pathologic lesions in rhesus monkeys exposed by aerosol. Technical Manuscript 226, US Government Printing Office, Washington, USA, pp.1-16.

25. Tseng J, Komisar JL, Chen JY, Hunt RE, Johnson AJ, et al. (1993) Immunity and Responses of Circulating Leukocytes and Lymphocytes in Monkeys to Aerosolized Staphylococcal Enterotoxin B. Infection and Immunity 6(2): 391-398.

26. Russell WMS, Burch RL, Hume (1959) CW: The Principles of Humane Experimental Technique. Methuen, London.

27. Flecknell P (2002) Replacement, reduction and refinement. Altex 19: 73-78

28. Vlach KD, Boles JW, Stiles BG (2000) Telemetric Evaluation of Body Temperature and Physical Activity as Predicators of Mortality in a Murine Model of Staphylococcal Enterotoxic Shock. Comparative Medicine 50(2): 160-166. 
29. Krakauer, Teresa, Marilyn Buckley (2012) Intranasal Rapamycin Rescues Mice from Staphylococcal Enterotoxin B-Induced Shock. Toxins 4(9): 718-728.

30. Roy, Chad J (2005) Human Leukocyte Antigen-DQ8 Transgenic Mice A Model To Examine the Toxicity of Aerosolized Staphylococcal Enterotoxin B. Infection and Immunity 73(4): 2452-2460.

31. Institue for Laboratory Animal Research. 2011. Guide for the care and use of laboratory animals, $\left(8^{\text {th }}\right.$ edn). National Academies Press, Washington, USA
32. United States Department of Agriculture (2013) Animal Welfare Act and Animal Welfare Regulations, U.S. Department of Agriculture, Animal and Plant Health Inspection Service, Washington, USA.

33. Laird NM, Ware JH (1982) Random-Effects Models for Longitudinal Data. Biometrics 38: 963-974.

34. Satterthwaite FE (1946) An Approximate Distribution of Estimates of Variance Components. Biometrics Bulletin 2: 110-114.

\section{Your next submission with Juniper Publishers will reach you the below assets}

- Quality Editorial service

- Swift Peer Review

- Reprints availability

- E-prints Service

- Manuscript Podcast for convenient understanding

- Global attainment for your research

- Manuscript accessibility in different formats

( Pdf, E-pub, Full Text, Audio)

- Unceasing customer service

Track the below URL for one-step submission https://juniperpublishers.com/online-submission.php 